\title{
O valor de um programa ludomotor para a melhoria da velocidade de reação de crianças
}

\section{The value of a ludomotor program for improving the reaction speed of children}

\author{
Alisson Padilha de Limal; Fabrício Bruno Cardoso2; Heron Beresford ${ }^{3}$; Iris Lima e Silva ${ }^{4}$ \\ ${ }^{1}$ Professor Bacharel e Licenciado em Educação Física - Centro Universitário Luterano de Ji-Paraná - CEULJI/ULBRA, Especialista \\ em Educação Especial - Universidade Católica Dom Bosco - UCDB/MS, Pesquisador do Laboratório de Temas Filosóficos em \\ Conhecimento Aplicado - LABFILC, Departamento de Ciências da Atividade Física - Universidade do Estado do Rio de Janeiro - \\ UERJ. Rio de Janeiro, RJ - Brasil. \\ ${ }_{2}^{2}$ Centro de Estudos em Neurociências e Educação (Neuroeduc/UFRJ), Programa de Neurobiologia do Instituto de Biofísica Carlos \\ Chagas Filho (IBCCF/UFRJ), Laboratório de Temas Filosóficos em Conhecimento Aplicado - LABFILC, Departamento de Ciências \\ da Atividade Física - Universidade do Estado do Rio de Janeiro - UERJ. Rio de Janeiro, RJ - Brasil \\ ${ }^{3}$ Professor Doutor em Filosofia, Adjunto da Universidade do Estado do Rio de Janeiro - UERJ, Coordenador de Pesquisas do \\ Laboratório de Temas Filosóficos em Conhecimento Aplicado - LABFILC, Departamento de Ciências da Atividade Física - \\ Universidade do Estado do Rio de Janeiro - UERJ. Rio de Janeiro, RJ - Brasil. \\ ${ }^{4}$ Departamento de Neurobiologia da Universidade Federal Fluminense - UFF, Laboratório de Temas Filosóficos em Conhecimento \\ Aplicado - LABFILC, Departamento de Ciências da Atividade Física - Universidade do Estado do Rio de Janeiro - UERJ. Rio de \\ Janeiro, RJ - Brasil. \\ Endereço para correspondência \\ Alisson Padilha de Lima, CREF: 001854 G/RO \\ R. Mogno, Bairro Setor 1, No 1980 \\ 76870-170 - Ariquemes - Rondônia \\ professor.alissonpadilha@gmail.com
}

\section{Resumo}

Objetivo: Avaliar a eficácia de um programa ludomotor de soltar pipas, voltado para desenvolver a velocidade de reação. Método: Este estudo foi desenvolvido utilizando-se os testes de reação motora (TRM), composto de três provas específicas: Tempo Reação Simples (TRS), Tempo de Reação com Discriminação (TRD) e Tempo de Reação com Discriminação para Tarefa Complexa (TRDTC). Resultados: Após 65 sessões do programa ludomotor, com duração de 45 minutos cada uma, mostrou-se, em relação ao TRS, TRD e TRDTC, uma significância estatística, em que foi identificada a importância da intervenção proposta. Conclusão: Verificou-se que a intervenção desenvolvida neste trabalho refinou as habilidades perceptivo-motoras dos alunos, o que se refletiu no desenvolvimento da coordenação visomotora.

Descritores: Crianças; Tempo de reação; Valor.

\begin{abstract}
Objective: To evaluate the efficacy of a "ludomotor" program involving flying kites, with the aim of developing reaction speed. Methods: This study involved the use of three motor reaction tests (MRTs): Simple Reaction Time (SRT), Reaction Time with Discrimination (RTD) and Reaction Time with Discrimination for Complex Tasks (RTDCT). Results: After 65 sessions of the "ludomotor" program with a duration of 45 minutes each, statistical significance was shown in relation to the SRT, MRT and RTDCT, showing the importance of the proposed intervention. Conclusion: It was demonstrated that the intervention designed in this study refined the perceptual motor abilities of the pupils, which was evidenced in the development of their visuomotor coordination.
\end{abstract}

Key words: Child; Reaction time; Value. 


\section{Introdução}

Cada vez mais focadas em estabelecer melhores níveis educacionais, as sociedades atuais buscam se servir das tecnologias de informação, direcionando metodologias pertinentes à função da capacitação do aluno para o processamento da aprendizagem ${ }^{1}$.

Quando se ensina algo, deseja-se que o indivíduo assimile a informação, retendo-a para uso posterior. A aprendizagem, neste caso, ocorre quando a pessoa consegue realizar uma atividade, como, por exemplo, elaborar operações matemáticas, andar de bicicleta ou nadar, mesmo após muitos anos sem praticá-la² .

A competência para processar as informações sensoriais emergentes do meio ambiente ou das percepções emanadas do próprio organismo do homem é um dos mais importantes fatores de natureza psicofisiológica que influencia no processo de aprendizagem, uma vez que o aperfeiçoamento dos mecanismos encarregados de operacionalizar tais informações se relaciona à habilidade para utilizar, de forma mais eficiente, a memória e as estratégias de processamento que evoluem paralelamente ao desenvolvimento perceptivo-motor experimentado pelo indivíduo $^{3}$. O uso gradativamente otimizado destes processos e estratégias, quando da operacionalização mental de tarefas motoras, reflete-se normalmente na eficiência com a qual estas tarefas são executadas ${ }^{4,5}$.

Essa otimização revela-se com a prática. A capacidade de seleção e retenção das informações específicas ou relevantes para a atividade que se realiza é nesta aperfeiçoada, o que poderá facilitar a antecipação da resposta e, consequentemente, melhorar a performance de uma pessoa ${ }^{6,7}$. Neste processo, a atenção exerce uma função muito importante, pois é por meio dela, associada aos mecanismos de controle, que se guardam as informações na memória de longa duração .

A atenção requer um estado de alerta e um adequado tônus cortical para a recepção dos estímulos que chegam pelos órgãos sensoriais, deste modo, a velocidade de processamento de informação aparece como indicador da capacidade intelectual dos indivíduos, sobretudo quando relacionada à execução de tarefas simples e complexas de reação, apontando, assim, para o tempo de reação ao estímulo como um método simples e eficiente para medir a atenção visual $^{9,10}$.

Justifica-se, assim, o objetivo deste estudo, isto é, avaliar a eficácia de um programa ludomotor de soltar pipas, voltado para desenvolver a velocidade de reação de crianças de seis a oito anos de idade, com dificuldade em realizar tarefas simples e complexas que exijam coordenação visomotora.

\section{Materiais e métodos}

Este estudo foi desenvolvido por meio de um formato ou desenho experimental, considerando-se que uma pesquisa experimental consiste em determinar um objeto de estudo, selecionar as variáveis que seriam capazes de influência-lo ${ }^{11}$.

O projeto deste trabalho foi submetido ao Comitê de Ética em Pesquisa da Universidade Castelo Branco (UCB/RJ) e aprovado sob o protocolo $\mathrm{n}^{\circ}$ 0001/2009. A coleta de dados se deu conforme a Resolução 196/96 do Conselho Nacional de Saúde do Ministério da Saúde. Assim, inicialmente foi solicitada a autorização dos pais ou responsáveis, por meio de um Termo de Consentimento Livre e Esclarecido, para que o estudo fosse desenvolvido.

Mediante avaliação clínica realizada por fonoaudiólogos especialistas em processos auditivos cerebrais, selecionaram-se os participantes que atenderam aos critérios de inclusão, ou seja, que não apresentaram disfunções neuromotoras de estrabismo e de déficits auditivos severos. Os sujeitos que não se enquadraram a esses critérios foram excluídos. Assim, formou-se um grupo de voluntários, tal como definido por Cochran ${ }^{12}$, composto por 50 alunos com idade compreendida entre seis e oito anos (média $=7,25$ anos), es- 
tudantes de uma escola particular da cidade do Rio de Janeiro, que tiveram frequência superior a $80 \%$ no processo de intervenção.

Todos os voluntários do estudo foram testados mantendo-se somente um aluno e o avaliador na sala de coleta dos dados (mesmo espaço utilizado por eles durante as aulas), com o objetivo de evitar qualquer tipo de perturbação. Ressalta-se que os participantes foram testados antes e após intervenção. O teste foi iniciado somente quando não havia mais dúvidas sobre o procedimento, não sendo realizada uma sessão de familiarização do aluno com aquele. $\mathrm{O}$ aluno permaneceu sentado em frente aos instrumentos de teste de reação motora (TRM) proposto por Morales ${ }^{13}$, composto de três provas específicas, quais sejam: Tempo Reação Simples (TRS), Tempo de Reação com Discriminação (TRD) e Tempo de Reação com Discriminação para Tarefa Complexa (TRDTC) ${ }^{13}$, em que foram preservados os mesmos avaliadores para todos os testes aqui examinados.

Para responder com precisão às provas, o estudante manteve o dedo indicador da mão de preferência, aquela utilizada para escrever, levemente apoiado sobre uma tecla de resposta (espaço). Os resultados obtidos nos Testes de Reação Motora (TRM) de cada aluno corresponderam à média dos 50 estímulos de cada prova.

São mostradas, a seguir, a ordem e a forma de aplicação das provas do TRM, estabelecendose um intervalo de cinco minutos entre cada uma delas:

1. Tempo Reação Simples (TRS) - o aluno deveria clicar sobre 50 figuras circulares (alvos verdadeiros) que apareciam individual e aleatoriamente no centro da tela, em espaço de até dois segundos entre cada aparição, tempo determinado pelo próprio software do teste.

2. Tempo de Reação com Discriminação (TRD) - o voluntário deveria clicar sobre 50 figuras quadriculares (alvos verdadeiros) que apareciam individual e aleatoriamente na lateral da tela, em espaço de até oito segundos entre cada aparição, tempo determinado pelo próprio software. Entre as aparições surgiam, também aleatoriamente, de uma a três figuras circulares (distratores).

3. Tempo de Reação com Discriminação para Tarefa Complexa (TRDTC) - exigiu que o aluno, após efetuação das respostas para as figuras quadriculares, pressionasse uma vez com o dedo indicador da mão utilizada para responder um interruptor de pressão interligado a um circuito elétrico sonoroluminoso localizado ao lado do laptop.

Foram considerados como erro os escores menores que 100 milésimos de segundos (ms), caracterizando uma possível antecipação da reação; e os maiores que $500 \mathrm{~ms}$, como algum problema de processamento do microcomputador.

A operacionalização de soltar pipas do programa ludomotor foi desenvolvida entendendose que a execução de tal ato motor exige de seu praticante atenção completas boas habilidades de memória, ligeira coordenação dos dedos e raciocínio rápido, além de percepção aguçada em face das adversidades, como perceber a mudança de direção do vento. O programa teve duração de 65 sessões, com 40 minutos cada uma, sendo realizada num espaço aberto de 25 metros quadrados, sendo em cada sessão evoluído o nível de exigências de manobras, partindo do simples para o complexo, em um período específico na parte da manhã para que não houvesse escores diferenciado nos testes.

Nas atividades, o manipulador recebia os comandos de velocidade e de mudanças de direção de um pesquisador, exigindo-se que o controle da pipa, ficasse dentro do limite dado, o que configurou um exercício de coordenação motora fina, que envolve um estado de atenção e concentração prolongado, para que fosse exercida uma plena organização dos movimentos e, principalmente, da coordenação visomotora.

A análise dos dados deste estudo foi realizada com base na comparação de resultados estatísticos utilizando-se o programa de estatística Bioestat 5.0, os resultados obtidos nas ava- 
liações do Tempo Reação Simples (TRS), Tempo de Reação com Discriminação (TRD) e Tempo de Reação com Discriminação para Tarefa Complexa (TRDTC) das crianças foram calculados por meio da estatística descritiva, a média e o desvio-padrão.

Para a comparação num sentindo pré-teste vs. pós-teste, os resultados obtidos foram testados para verificar sua normalidade pelo teste de Shapiro-Wilk, sendo classificado, como paramétrico, os resultados obtidos em relação ao TRS e TRD; e como não paramétrico, os adquiridos em relação ao TRDTC.

De acordo com os resultados obtidos no teste de normalidade, optou-se pelos testes " $\mathrm{t}$ " de Student, para comparação entre as avaliações acerca do TRS e TRD; e de Wilcoxon, para comparação em relação ao TRDC. Cabe ressaltar que, em ambas as comparações, foi estabelecido o nível de significância de 5,00\%, o que configura uma certeza mínima na tomada de decisão de $95,00 \%$.

\section{Resultados}

A partir da Figura 1, observou-se que, referente ao TRS, os alunos apresentaram, no instante pré-teste, uma variação de resultados entre 273 e 321 ms, com desvio-padrão de 11,95 ms, média de $290,84 \mathrm{~ms}$ e um coeficiente de variação de $4,11 \%$. Já no instante pós-teste, a variação de resultados ficou entre 258 e 312 ms, com desvio-padrão de 10,92, pois a média caiu para 273,7 , e um coeficiente de variação igual a 3,99\%. Cabe ressaltar que quanto menor for o tempo de resposta ao estímulo, melhor o desempenho do aluno. Utilizando o teste " $\mathrm{t}$ " para duas amostras pareadas foi possível analisar estatisticamente os efeitos positivos da intervenção desenvolvida neste estudo em relação ao TRS, uma vez que se revelou, para um $\mathrm{t}=14,71$, um $\mathrm{p}=0,01$.

Conforme mostra Figura 2, quando avaliados no instante pré-intervenção em relação ao TRD, os alunos apresentaram resultados variando entre 317 e 402 ms, um desvio-padrão de

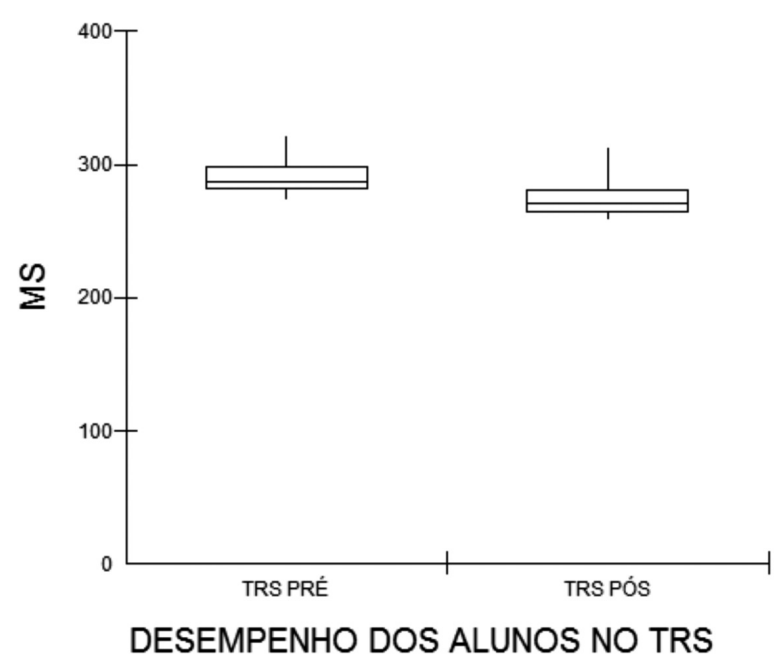

Figura l: Desempenho das crianças no TRS

14,65 e uma média de 342,26 ms, com coeficiente de variação igual a 4,28\%. Já no instante pós, esta foi $2,63 \%$, pois os resultados ficaram entre 309 e 342 ms, o desvio-padrão em 8,49, e a média em 332,42 ms, o que revela tendência a um efeito positivo da atividade motora em soltar pipa sobre a capacidade dos alunos em responder de maneira rápida a estímulos próprios de uma tarefa que necessite de coordenação visomotora. A comprovação dos efeitos positivos da intervenção sobre o TRD dos alunos participantes deste estudo se deu também por meio do teste " $\mathrm{t}$ " para duas amostras pareadas, resultando em $\mathrm{t}=11,98 \mathrm{e}$ um $\mathrm{p}=0,02$.

Na Figura 3 é possível observar que, inicialmente, os alunos apresentaram variação entre 324 e 367 ms para responder ao estímulo dado, com desvio-padrão de 9,47, média de 343,86 ms e coeficiente de variação de $2,76 \%$, em relação ao TRDTC. Já na segunda avaliação, a variação de resultados ficou entre 318 e 352 ms, com um desvio-padrão de 8,13, uma média que caiu para $331,62 \mathrm{~ms}$, revelando coeficiente de variação de $2,45 \%$.

Através do teste de Wilcoxon para duas amostras pareadas foi possível comprovar a melhora do processamento seletivo motor dos alunos ao responder corretamente a uma tarefa motora complexa em um curto espaço de tempo, sendo identificado um $\mathrm{p}=0,01(\mathrm{Z}=6,15)$. 


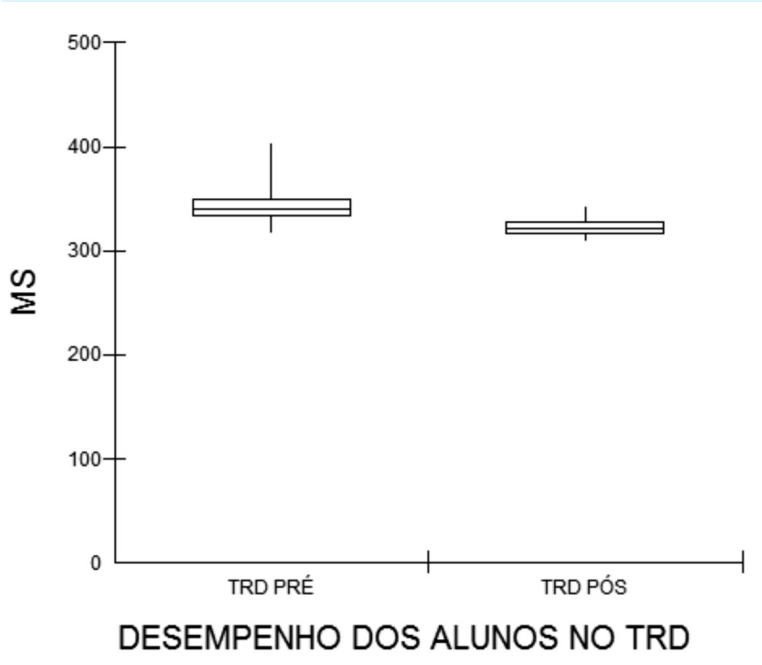

Figura 2: Desempenho das crianças no TRD

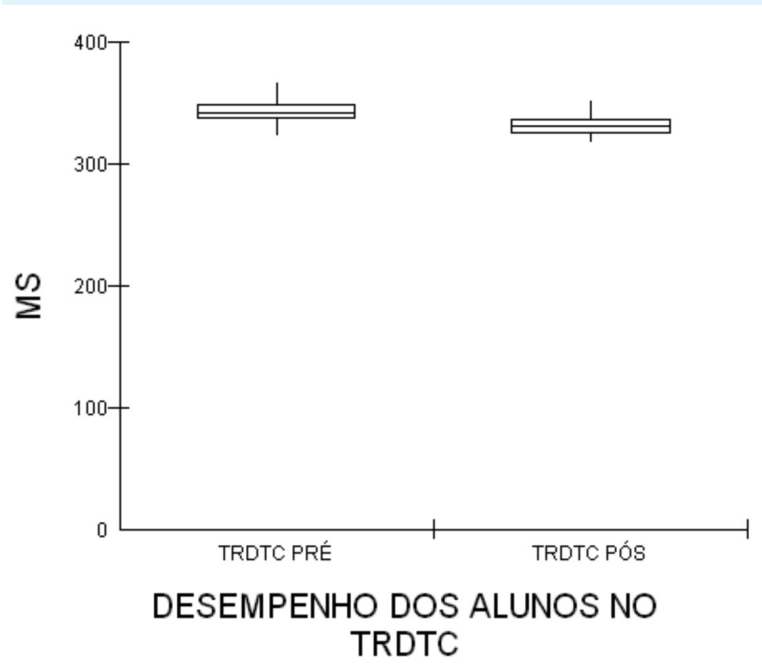

Figura 3: Desempenho das crianças no TRDC

\section{Discussão}

Partindo do pressuposto que o Tempo de Reação é um indicador da velocidade de processamento da informação, a melhora no TRS apresentada pelos alunos pode ter ocorrido pelos efeitos da intervenção desenvolvida neste estudo em sua performance motora, pois a referida intervenção proporcionou aos participantes melhor capacidade na organização dos estímulos visuais, auditivos, táteis e vestibulares por eles percebidos ${ }^{2,3}$. Assim, acredita-se nisso, porque os processos fisiológicos de transformação dos estímulos auditivos e visuais em resposta motora são idênticos, em todas as estimulações de desenvolvimento motor, estando o diferencial no produto final, que é a forma de se atribuir a mecânica do movimento, ou seja, de se fazer pensar nos grupamentos musculares que são recrutados para realizar determinadas tarefas ${ }^{5,6}$.

Por meio dos escores, sugere-se a existência da relação da melhoria das funções cognitivas com um maior tempo de prática de atividades motoras. Isto é conseguido pela única etapa treinável (tempo de elaboração da resposta) envolvida do sistema nervoso num processo de tomada de decisão cognitiva e perceptiva durante a preparação do movimento em tarefas de reações complexas ${ }^{14}$.

Em outra intervenção motora realizada por intermédio do judô, atividade diferente da utilizada neste estudo, obteve-se escores significativos no tempo de reação das crianças avaliadas, possibilitando, assim, uma melhora no desempenho motor mediante uma melhor capacidade de seleção e elaboração da resposta durante a execução do ato motor ${ }^{15}$.

Já em outra intervenção esportiva os pesquisadores Ruschel et al. ${ }^{16}$ observaram escores significativos no tempo de reação simples de jogadores de futebol e identificaram a importância de executar intervenções que proporcionem melhoras no repertório motor e no TR, o que vem a corroborar a pesquisa aqui apresentada, em que se identificaram melhoras nos escores do TR pela intervenção ludomotora.

Portanto, a escolha da intervenção motora é primordial para a melhora das variáveis analisadas, como o tempo de reação. Pode-se observar isso, em um estudo com intervenção eficaz por meio da ginástica artística, no qual os autores Duarte et al. ${ }^{17}$ não obtiveram escores positivos, e puderam verificar, assim, a impossibilidade de uma melhora na performance, em razão de uma menor capacidade de reação, comprovando ser um método ineficaz sobre o TR.

Os resultados sugerem que a estimulação ludomotora de soltar pipa facilitou o desenvolvi- 
mento da capacidade dos alunos em relação à interceptação de objetos ou cálculo do timing com antecipação, fazendo com que sejam capazes de combinar as estimativas da localização de um objeto com uma reação motora específica ${ }^{18}$.

Dessa forma, o tempo de reação, quando esta é bem estimulada, pode influenciar na capacidade de o sujeito responder de forma veloz a um estímulo, bem como contribuir na seleção de novos talentos esportivos, por meio da variável estratificada tempo de reação cognitivo que representa a qualidade da percepção de um indivíduo ter a condição de ser um atleta ou não ${ }^{19}$. Assim, pode-se constatar o valor da contribuição da intervenção ludomotora no sistema motor das crianças avaliadas neste estudo. Isto porque, entende-se por valor uma qualidade estrutural de natureza metafísica que corresponde a tudo aquilo (no caso, o referido programa ludomotor de soltar pipas) que preenche positivamente um complexo estado de carência, privação ou de vacuidade - sendo, nesse contexto, a dificuldade em realizar tarefas simples e complexas e que exijam coordenação visomotora de seres humanos (as crianças participantes deste estudo) ${ }^{20}$.

\section{Conclusões}

Pôde-se concluir que a intervenção desenvolvida neste estudo refinou as habilidades perceptivo-motoras e a velocidade de processamento de informação dos alunos, definindo melhor suas dimensões temporais, o que se refletiu no desenvolvimento da coordenação visomotora observada nos resultados finais da pesquisa. Assim, as crianças aqui avaliadas passaram a desempenhar tarefas complexas com maior facilidade e em curto espaço de tempo.

Portanto, acredita-se que os participantes deste estudo apresentaram uma melhora no mecanismo de decisão logo após receber a transmissão das informações processadas pelo mecanismo perceptivo e de escolher o plano motor adequado para a realização da tarefa proposta, levando em consideração o meio ambiente e os objetivos originais da performance - o que significa melhorar a capacidade de um indivíduo para a compreensão total da habilidade que a tarefa exige -, confirmando o valor ou os efeitos positivos que o programa ludomotor de soltar pipas podem trazer aos indivíduos submetidos a este tipo de intervenção.

Recomenda-se que sejam feitas mais pesquisas avaliando a prática ludomotora de soltar pipas, como intervenção eficaz de ganhos e melhoras no sistema perceptivo motor de crianças de ambos os sexos.

\section{Referências}

1. Burns OM, Rajan R. Learning in a task of complex auditory streaming and identification. Neurobiol Learn Mem. 2008;89:448-61.

2. Chang LY, Klatzky RL, Pollard NS. Selection criteria for preparatory object rotation in manual lifting actions. J Mot Behav. 2010;42(1):11-27.

3. Ford P, Nicolas J, Huys R, Williams AM. An evaluation of end-point trajectory planning during skilled kicking. Motor Control. 2009;13:1-24.

4. Lipski SC, Unger S, Grice M, Meister IG. Masked auditory feedback affects speech motor learning of a plosive duration contrast. Motor Control. 2011;15:68-84.

5. Silva VF, Poly MWO, Ribeiro Junior SMS, Calomeni MR, Pinto MVM, Silva ALS. Efeito agudo da estimulação cerebral, através de luz e som, no tempo de reação motora de jovens atletas. Revista Digital de Deportes, Buenos Aires, 2008;13(120):1-1.

6. Piquard A, Lacomblez L, Derouesné C, Siéroff E. Problems inhibiting attentional capture by irrelevant stimuli in patients with fronto temporal dementia. Brain Cogn. 2009;70:62-6.

7. Marigold DS, Weerdesteyn V, Patla AE, Duysens J. Keep looking ahead? Redirection of visual fixation does not always occur during an unpredictable obstacle avoidance task. Exp Brain Res. 2007;176:32-42.

8. Moraes R, Allard F, Patla AE. Validating determinants for alternate foot placement selection algorithm during human locomotion in a cluttered terrain. J Neurophys. 2007;98:1928-40. 
9. Siéroff E, Decaix C, Chokron S, Bartolomeo P. Impaired orienting of attention in left unilateral neglect: a componential analysis. Neuropsychology. 2007;21:94-113.

10. Corradeschi F. Attentional in athletes of high and low experience engaged in different open skill sports. Percept Mot Skills. Missoula, 2007;102:791- 805 .

11. Thomas RJ, Nelson JK, Silverman SJ. Métodos de pesquisa em atividade física. Tradução Sales DR. Dornelles MS. 5. ed. Porto Alegre: Artmed; 2007.

12. Cochran G. Tecnologia da amostragem. Rio de Janeiro: Fundo de Cultura; 1956.

13. Morales VAM, Silva VF. Desenvolvimento cognitivo com aspectos no voleibol. Tecnologia Educacional, Rio De Janeiro, 2006;167(16):89-98.

14. Rodrigues RB, Postai E, Katzer JI, Palma LE, Corazza ST. Tempo de reação e equilíbrio de escolares com e sem professor de educação física nas séries iniciais. Pensar Prát. 2011;14(2):1-15.

15. Lima VF, Oliveira FF, Sinésio T, Vieira MM. Efeitos da prática sistemática do judô no tempo de reação de crianças. Pensar Prát. 2011;14(1):1-9.
16. Ruschel C, Haupenthal A, Hubert M, Fontana HB, Pereira SM, Roesler H. Tempo de reação simples de jogadores de futebol de diferentes categorias e posições. Motricidade. 2011;7(4):73-82.

17. Duarte AA, Santos FCA, Barbosa CC, Vieira MM. O efeito da prática sistemática da ginástica artística no tempo de reação de crianças. Revista Digital de Deportes, Buenos Aires, 2011;16(161):1-1.

18. Aleixo IMS, Teixeira FAC, Vieira MM. Relação entre tempo de reação e tempo de movimento em uma habilidade motora com crianças. Motricidade. 2012;8(2):1032-7.

19. Noce F, Ferreira TS, Moreira CZ, Andrade AGP, Mello MT, Costa VT. Influência do tempo de reação simples na seleção de jovens talentos no tênis. Rev Educ Fis/UEM, 2012;23(3):369-77.

20. Beresford H, Rosa AS, Rosa TS, Cardoso FB. O valor da informática educacional para a melhora do desenvolvimento lógico-matemático de crianças. Meta: Avaliação. Rio de Janeiro, 2010;2(4):60-72. 